\title{
Comparison of clinical and neuropathological diagnoses of neurodegenerative diseases in two centres from the Brains for Dementia Research (BDR) cohort
}

\author{
Sashika Selvackadunco ${ }^{1}$ (D) $\cdot$ Katie Langford ${ }^{2} \cdot$ Zohra Shah $^{1} \cdot$ Siobhan Hurley $^{2} \cdot$ Istvan Bodi $^{1,3} \cdot$ Andrew King $^{1,3}$. \\ Dag Aarsland ${ }^{2}$. Claire Troakes ${ }^{1} \cdot$ Safa Al-Sarraj ${ }^{1,3}$
}

Received: 28 September 2018 / Accepted: 15 December 2018 / Published online: 7 February 2019

(c) The Author(s) 2019

\begin{abstract}
Early detection and accurate diagnosis of neurodegenerative disorders may provide better epidemiological data, closer monitoring of disease progression and enable more specialised intervention. We analysed the clinical records and pathology of brain donations from 180 patients from two Brains for Dementia Research cohorts to determine the agreement between in-life clinical diagnosis and post-mortem pathological results. Clinical diagnosis was extracted from medical records and cases assigned into broad clinical groups; control, Alzheimer's disease (AD), vascular dementia (CVD), dementia with Lewy bodies (DLB), frontotemporal dementia (FTD) and combined diseases. Pathology was assessed blindly, and cases categorised into; control, intermediate $\mathrm{AD}$, severe $\mathrm{AD}, \mathrm{CVD}, \mathrm{AD}$ and CVD combined, $\mathrm{DLB}, \mathrm{AD}$ and DLB combined and frontotemporal lobar degeneration (FTLD), according to the major contributing pathologies. In more than a third of cases clinical diagnosis was different from final neuropathological diagnosis. The majority of AD, DLB and control clinical groups matched the pathological diagnosis; however, thirty-five percent of clinical AD cases showed additional prominent CVD or DLB pathology which had not been diagnosed clinically and twenty-five percent of clinical control cases were found to have intermediate Tau pathology (modified Braak stage III-IV) or CVD. CVD and AD + CVD clinical groups showed an average of only thirty-two percent pathological correlation, the majority actually having no CVD, and fifty-three percent of pathologically identified FTLD cases had been incorrectly clinically diagnosed. Our results underlie the importance of neuropathological confirmation of clinical diagnosis. The relatively low accuracy of clinical diagnosis demonstrates the need for standardised and validated diagnostic assessment procedures.
\end{abstract}

Keywords Brain banking - Neuropathology · Brain donation - Brains for Dementia Research · Clinical diagnosis . Clinicopathological correlation

Safa Al-Sarraj

safa.al-sarraj@nhs.net

1 Department of Basic and Clinical Neuroscience, IoPPN, King's College London, London, UK

2 Old Age Psychiatry Department, IoPPN, King's College London, London, UK

3 Department of Clinical Neuropathology, Academic Neuroscience Centre, King's College Hospital, King's College Hospital NHS Foundation Trust, 1st Floor, Denmark Hill, London SE5 9RS, UK

\section{Introduction}

Research shows that most people currently living with dementia have not received a formal diagnosis, with only $20-50 \%$ of dementia cases in high-income countries being formally recognised and documented in primary care settings (Alzheimer's Disease International World Alzheimer Report 2011). This number is much greater in low- and middle-income countries.

Medical interventions and therapies are only available for those that have sought and received a diagnosis. Researchers are developing new drugs that may slow or stop the progression of the disease, particularly in the preclinical/early stage; however, trials of potentially preventative agents will only be possible once reliable methods of early diagnosis have 
been established. Increased reliability of diagnosis would also allow for the progression of the disease to be monitored more closely and provide an opportunity for more accurate determination of the specific dementia subtype. This would enable more specialised intervention and treatment at an earlier stage of the disease, before extensive neurochemical and neuropathological changes have occurred.

As $50 \%$ of people with mild cognitive impairment (MCI) later develop dementia, this would be a crucial group to monitor. The National Institute for Health and Care Excellence (NICE 2006) guidelines urge primary healthcare staff to refer people who show signs of MCI to memory assessment services and when these services identify people with MCI (including those without memory impairment) they are advised to offer followup assessments to monitor cognitive decline. To improve the accuracy of the diagnosis made, NICE guidelines suggest a diagnosis of dementia should only be made after a comprehensive assessment has taken place (NICE 2018). Healthcare professionals with expertise in differential diagnosis are encouraged to use international standardised criteria to obtain a more specific subtype of dementia diagnosis, they suggest encompassing various structural imaging techniques to aid in differentiation when appropriate (NICE 2006, 2018).

Dementia has many causes, the most common type being Alzheimer's disease (AD), followed by vascular dementia (CVD), and dementia with Lewy bodies (DLB), then less common types such as frontotemporal dementia (FTD) and others. Though the guidelines provide much hope for better characterised diagnosis of dementia in the future, currently terms such as unspecified dementia or mixed AD and vascular dementia are commonly used, which clinically describe a wide range of symptoms associated with a decline in memory and cognitive function.

Whilst "dementia" can only be diagnosed clinically the subtypes, including Alzheimer's disease, dementia with Lewy bodies and vascular dementia are clinicopathological entities requiring certain pathological characteristics for definitive diagnosis. The well-defined guidelines for such diagnoses include the BNE modified Braak score (Braak 2006), NIA-ABC Scoring (Alafuzoff 2009), McKeith Lewy body disease Score (McKeith 2005), and VCING (Skrobot 2016). However, without an accurate in-life clinical diagnosis and assessment data to validate the pathological findings we still have an incomplete picture of the disease.

Projects such as Brains for Dementia Research (BDR) have the unique quality of providing longitudinal clinical data alongside post-mortem brain tissue. BDR is a network of brain bank centres across England and Wales established in 2007. Through regular standardised clinical assessments, such as mini mental state examination (MMSE), participants are monitored to assess memory, lifestyle and behavior. BDR participants are assessed annually if they have a memory impairment or diagnosis of dementia, and every 2 years if they are a healthy control (Hayes 2016). The assessments do not contribute to any formal clinical diagnosis.

Once the participant has passed away the brain tissue is donated to BDR associated brain banks who are able to establish the post-mortem diagnosis; both clinical assessment details and medical notes contribute to the eventual neuropathological diagnosis. The pathological evaluations for both London and Cardiff BDR cohorts are carried out in the neuropathology department of King's College London.

This study analysed the BDR cohort from the London and Cardiff centres to determine the agreeability and discrepancy between in life clinical diagnosis and eventual pathological results; and examine the reliability of GP/memory clinic diagnosis of dementia alongside the efficiency of BDR clinical assessments. The results may aid in future clinical assessments and interpretation of symptoms to enable more accurate and specific diagnoses of dementia types.

\section{Materials and methods}

Consent for clinical assessment, autopsy, neuropathological assessment and research were obtained from all subjects and all studies were carried out under the ethical approval of the MRC London Neurodegenerative Diseases Brain Bank and the Brains for Dementia Research project. All BDR cases from the London and Cardiff cohorts received between 2009 and 2016 were reviewed. The clinical diagnosis of each participant and where the diagnosis was made (i.e., hospital, GP, memory clinic) was extracted from the medical records. The most recent BDR clinical assessment scores for each case were also examined.

\section{Clinical diagnosis}

The vast majority of dementia cases were given a diagnosis by a hospital team (Table 1). As they were assessed in secondary care it can reasonably be presumed that NICE

Table 1 Origin of dementia diagnosis

\begin{tabular}{lcl}
\hline Origin of dementia diagnosis & Frequency & Percent \\
\hline $\begin{array}{l}\text { No dementia diagnosis given-clinical } \\
\text { control }\end{array}$ & 56 & $\mathrm{n} / \mathrm{a}$ \\
GP & 6 & 4.8 \\
Hospital team & 84 & 67.2 \\
Memory service & 20 & 16 \\
Older adult MH & 5 & 4 \\
Unknown/unspecified & 10 & 8 \\
Total & 180 & 100.0 \\
\hline
\end{tabular}


approved procedures were followed and that they received specialised assessments following referral from a GP.

The 180 cases were grouped into 8 broad clinical groups by the BDR study clinical co-ordinators (DA, SH) who were blind from any neuropathological findings: (1) control: no dementia diagnosis or evidence of cognitive change; (2) AD: Alzheimer's disease diagnosis including late onset, early onset, unspecified AD; (3) CVD: vascular dementia or cerebrovascular disease (including small vessel disease); (4) AD + CVD: Alzheimer's disease and vascular features described; (5) DLB: dementia with Lewy bodies; (6) AD and DLB: Alzheimer's disease with additional features of dementia with Lewy bodies; (7) FTD: frontotemporal dementia; and (8) other or unspecified: e.g., paranoid schizophrenia, dementia without specified type.

Neuropathological findings were analysed and broadly grouped by neuropathologists (SAS, IB) who reviewed the microscopy reports of all cases independent of any clinical detail. Cases were categorised into specific groups depending on the type and degree of contributing pathologies present. Modified Braak score, NIA-ABC and CERAD score, VCING and McKeith Lewy body disease criteria were analysed to prioritise the pathological findings and correctly group the cases (Table 2).

Once all cases were grouped according to their clinical and pathological diagnoses, both data sets were combined. Each case was assessed to determine if the clinical diagnosis matched with the final neuropathological findings.

If the clinical and pathological group for a case did not match, the case was reviewed to establish whether this mismatch could be explained; the pathological reports were revisited in detail, as well as GP notes and further assessment records. This resulted in some originally nonmatched cases subsequently being determined to match, based on clinical circumstances (e.g., pathology was a sudden event just before death) or accounting for factors such as age-related changes, which the pathology scores alone did not consider. Within the largest clinical groups (control

Table 2 Outline of the pathological groups and definitions

\begin{tabular}{|c|c|c|}
\hline Group & Description & Pathology score \\
\hline CONTROL & No pathology, low or age-related pathology, and/or other minor pathology & $\begin{array}{l}\text { BRAAK (BNE) Score 0-II } \\
\text { CERAD Not AD-Low } \\
\text { VCING No CVD-Low }\end{array}$ \\
\hline IM AD: intermediate & $\begin{array}{l}\text { Alzheimer's disease type changes may or may not indicate cognitive decline; includ- } \\
\text { ing those with secondary pathology e.g. amyloid angiopathy, TDP- } 43 \text { pathology }\end{array}$ & $\begin{array}{l}\text { BRAAK (BNE) Score III-IV } \\
\text { CERAD Moderate-High } \\
\text { VCING No CVD-Moderate }\end{array}$ \\
\hline AD: severe & $\begin{array}{l}\text { Severe Alzheimer disease changes indicating cognitive decline; including those with } \\
\text { secondary pathology e.g. amyloid angiopathy, TDP- } 43 \text { pathology }\end{array}$ & $\begin{array}{l}\text { BRAAK (BNE) Score V-VI } \\
\text { CERAD Moderate-High } \\
\text { VCING No CVD-Moderate }\end{array}$ \\
\hline CVD & Cerebrovascular disease, including small vessel disease & $\begin{array}{l}\text { VCING Moderate-High } \\
\text { BRAAK (BNE) Score 0-II } \\
\text { CERAD Not AD-Low }\end{array}$ \\
\hline $\mathrm{AD}+\mathrm{CVD}$ & $\mathrm{AD}$ and significant CVD pathology & $\begin{array}{l}\text { BRAAK (BNE) III-VI* } \\
\text { CERAD Intermediate-High } \\
\text { VCING Moderate-High }\end{array}$ \\
\hline DLB & Dementia with Lewy Bodies; in absence of AD pathology & $\begin{array}{l}\text { McKeith Limbic/ Neocortical } \\
\text { stage } \\
\text { DLB Braak stage: } 5-6 \\
\text { Prob of DLB: Moderate-High } \\
\text { BRAAK (BNE) 0-II }\end{array}$ \\
\hline $\mathrm{AD}+\mathrm{DLB}$ & AD and significant Lewy body pathology & $\begin{array}{l}\text { McKeith Limbic/ Neocortical } \\
\text { stage } \\
\text { DLB Braak stage 5-6 } \\
\text { Prob of DLB Moderate-High } \\
\text { BRAAK (BNE) III-VI* } \\
\text { CERAD Moderate-High }\end{array}$ \\
\hline FTLD & $\begin{array}{l}\text { Combined all frontotemporal lobar degeneration types, including **CBD, PSP and } \\
\text { globular glial tauopathy (GGT) }\end{array}$ & Any FTLD subtypes \\
\hline Other & $\begin{array}{l}\text { Other diagnosis such as Argyrophillic grain disease (AGD), herpes simplex virus } \\
\text { (HSV-1) and infarcts }\end{array}$ & \\
\hline
\end{tabular}

Neuropathological groups: *When AD is mentioned combined with CVD or DLB it includes both intermediate and severe AD pathology

**CBD and PSP were included in the FTLD pathological group- as although clinical presentation of these conditions could include Parkinsonian features there was no mention of these syndromes in the medical notes 
and $\mathrm{AD}$ ) the BDR assessment scores were statistically analysed using an independent sample $T$ test to identify any trends or significant differences in values between matching and non-matching cases.

\section{Results}

\section{Correlation between clinical and neuropathological diagnosis}

Clinical and neuropathological diagnosis was determined to match in only 115 of the 180 cases examined (Fig. 1). Only 37 out of the 75 clinical AD cases were a direct match pathologically, with most of these cases being classified as severe AD (modified Braak stage V-VI), a further
26 of these clinical AD cases had coexisting DLB or CVD pathology that had not been detected clinically (Table 3). Seven of the clinical AD cases were determined pathologically to be FTLD. The CVD and AD + CVD clinical groups had a wider range of different pathological diagnoses, though many of these had either severe AD pathology or mixed pathology of either AD + CVD or AD + DLB.

The majority (3/4) of the cases with a clinical DLB diagnosis were matched with AD + DLB pathology, with the remaining case being diagnosed as AD. In the clinical FTD group, four out of six of the cases were pathologically matched with FTLD, the other two cases were diagnosed as AD+DLB and AD + CVD. Within the clinical control group, 40 out of the 55 participants were determined to be controls pathologically (i.e., no or very minor pathology present); however, the remaining 15 cases were found to have

Fig. 1 The number of matching and non-matching cases in each clinical group

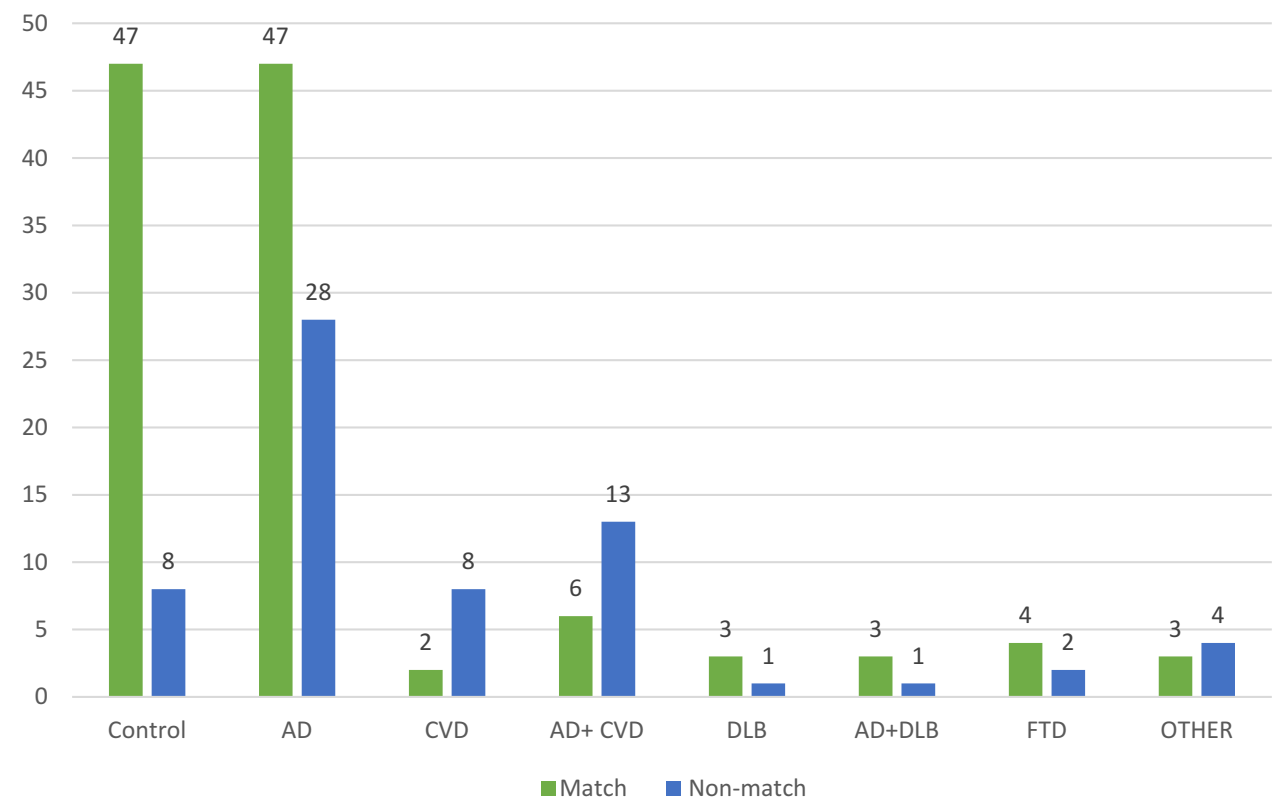

Table 3 Cross tabulation of clinical diagnosis and final neuropathological diagnosis

\begin{tabular}{|c|c|c|c|c|c|c|c|c|c|c|c|c|}
\hline & & & Patholo & cal Diag & & & & & & & & \\
\hline & & $\begin{array}{l}\text { range } \\
\text { (years) }\end{array}$ & Control & IM-AD & $\begin{array}{l}\text { Severe } \\
\text { AD }\end{array}$ & CVD & $\begin{array}{l}\mathrm{AD}+ \\
\mathrm{CVD}\end{array}$ & DLB & $\begin{array}{l}\mathrm{AD}+ \\
\mathrm{DLB}\end{array}$ & FTLD & OTHER & Total \\
\hline & Control & $59-103$ & 40 & 9 & 0 & 3 & 1 & 0 & 0 & 1 & 1 & 55 \\
\hline & $\mathrm{AD}$ & $62-99$ & 1 & 3 & 34 & 2 & 14 & 1 & 12 & 7 & 1 & 75 \\
\hline Clinical & CVD & 79-95 & 0 & 2 & 0 & 1 & 3 & 1 & 2 & 0 & 1 & 10 \\
\hline & $\mathrm{AD}+\mathrm{CVD}$ & $66-92$ & 0 & 0 & 6 & 1 & 5 & 0 & 5 & 2 & 0 & 19 \\
\hline & DLB & $82-92$ & 0 & 1 & 0 & 0 & 0 & 0 & 3 & 0 & 0 & 4 \\
\hline & $\mathrm{AD}+\mathrm{DLB}$ & $74-83$ & 0 & 0 & 1 & 0 & 0 & 0 & 3 & 0 & 0 & 4 \\
\hline & FTD & $70-83$ & 0 & 0 & 0 & 0 & 1 & 0 & 1 & 4 & 0 & 6 \\
\hline & OTHER & $81-93$ & 0 & 0 & 1 & 2 & 1 & 0 & 2 & 1 & 0 & 7 \\
\hline Total & & & 41 & 15 & 42 & 9 & 25 & 2 & 28 & 15 & 3 & 180 \\
\hline
\end{tabular}


significant pathology despite no report of cognitive decline (Table 3).

The number of matching and non-matching cases in each clinical group was recalculated after further review of the cases to account for any explanation of the mismatch (see "Materials and methods") and the pathology groups were reorganised by splitting cases from the intermediate $A D$ group into either control and $\mathrm{AD}$ depending on the whether the pathology present was deemed to be normal age-related changes, or indication of Alzheimer's disease progression. Percentage matches within each of the clinical diagnosis groups were then calculated (Table 4), although we excluded the 'other' group from further analysis due to the diversity of diagnosis within the group and the inability to match these appropriately.

Overall $64 \%$ of all cases were deemed to match clinically and pathologically. The control, AD, DLB, AD + DLB and FTD clinical groups had a majority of cases matching; however, the CVD and AD + CVD group were poorly matched with only $20-32 \%$ of the cases matching.

\section{Clinical assessment results}

Table 5 shows 123 cases, where MMSE assessments had been carried out within 4 years prior to death. Based on NICE guidelines BDR considers a score of nine or under as showing severe impairment of cognitive function, 10-20 as moderate impairment of cognitive function, 21-24 as mild impairment of cognitive function and a score of 25 to the maximum 30 would be considered normal (NICE 2001).

Table 6 shows the spread of the most recent MMSE scores between diagnosis groups. As not all the assessments taken had been scored up to the full 30 points (e.g., patient was unable to complete all the tasks), percentage scores were used to group the cases between cognitive states. Normal (> 83\%), mild impairment of cognitive function (70-82\%), moderate impairment of cognitive function (33-69\%), and severe impairment of cognitive function $(<32 \%)$. The

Table 4 Percentage of match and non-matched cases within each clinical diagnosis groups

\begin{tabular}{lll}
\hline Clinical diagnosis & Matched $(\%)$ & $\begin{array}{l}\text { Non- } \\
\text { matched } \\
(\%)\end{array}$ \\
\hline Control & 85.5 & 14.5 \\
AD & 62.7 & 37.3 \\
CVD & 20.0 & 80.0 \\
AD+CVD & 31.6 & 68.4 \\
DLB & 75.0 & 25.0 \\
AD+DLB & 75.0 & 25.0 \\
FTD & 66.7 & 33.3 \\
Total & 64.4 & 35.6 \\
\hline
\end{tabular}

control group, as expected, had the highest mean score for MMSE. The mean scores for FTD and AD cases were significantly lower than all other conditions which suggests the MMSE scoring system may be more able to identity FTD and $\mathrm{AD}$, and that diagnosis of these conditions may be aided by the more obvious presentation of cognitive decline via MMSE assessments.

An independent sample $T$ test was carried out on the clinical 'control' group and clinical 'AD' group to determine if there was any significant difference between the matched and non-matched cases, and to identify any trends in the BDR Clinical assessments (MMSE, NPI, GDS, CDR scores) across the groups. No significant difference was found in any of the clinical assessment scores between the matched and non-matched cases in either clinical group.

\section{Discussion}

Analysis of the diagnosis in the London and Cardiff BDR cohort demonstrates that there are a large proportion (36\%) of cases that show a different pathological diagnosis to the one given clinically.

$25 \%$ (14 out of 55) of the clinical control group exhibited significant pathology. If these cases were in the preclinical phase of AD, as describe by Jack et al. (2011), presentation of clinical symptoms may have been absent. The cases could alternatively have been in the pre-dementia or MCI phase of AD, as described by Sperling et al. (2011), and any symptoms presented too subtle to indicate a dementia diagnosis. This is supported by the BDR clinical assessment data, which did not show any significant difference in assessment scores between matched and non-matched controls.

Age is vital to consider when diagnosing a patient with AD. Price et al. (2009) examined a group of cognitively normal people and found that neuropathological processes related to $\mathrm{AD}$ in persons without dementia appear to be associated with subtle cognitive dysfunction representing a preclinical stage of the illness (2009). Interestingly, by the age of 80-85 years, as many as $40 \%$ of non-demented cases demonstrated AD pathology. With strict criteria applied approximately $20 \%$ of cases had AD pathology. In our study $16 \%$ (9 out of 55) of clinical controls had moderate AD pathology (i.e., modified Braak stages III-IV); up to $20 \%$ when the 3 CVD and 1 FTLD cases are included, which had accompanying Braak stage III-IV pathology.

During the re-analysis, the neuropathologists determined that in five out of the nine cases originally scored as modified Braak stage III-IV there had been artificial elevation of the apparent modified Braak stage by actual age-related changes (Crary 2014) which would not have presented as clinical dementia; these cases were, therefore, no longer considered to be mismatched. The other four cases were not 
Table 5123 of the 180 cases with recent MMSE scores

\begin{tabular}{|c|c|c|c|c|c|c|c|c|}
\hline MRC ID & Age at death & Gender & MMSE score & MMSE out of & $\begin{array}{l}\text { Percentage } \\
\text { score }(\%)\end{array}$ & $\begin{array}{l}\text { Coded } \\
\text { MMSE }\end{array}$ & $\begin{array}{l}\text { Impairment of } \\
\text { cognitive func- } \\
\text { tioning }\end{array}$ & Clinical Diagnosis Group \\
\hline BBN_25640 & 95 & $\mathrm{~F}$ & 24 & 26 & 92.31 & 0 & Normal & CONTROL \\
\hline BBN002.26316 & 96 & $\mathrm{~F}$ & 25 & 30 & 83.33 & 0 & Normal & CONTROL \\
\hline BBN002.29633 & 96 & $\mathrm{~F}$ & 26 & 30 & 86.67 & 0 & Normal & CONTROL \\
\hline BBN_24297 & 83 & $\mathrm{~F}$ & 27 & 30 & 90.00 & 0 & Normal & CONTROL \\
\hline BBN002.30036 & 89 & $\mathrm{~F}$ & 28 & 30 & 93.33 & 0 & Normal & CONTROL \\
\hline BBN_21005 & 77 & $\mathrm{~F}$ & 28 & 30 & 93.33 & 0 & Normal & CONTROL \\
\hline BBN002.29730 & 91 & $\mathrm{~F}$ & 28 & 30 & 93.33 & 0 & Normal & CONTROL \\
\hline BBN_10199 & 93 & $\mathrm{~F}$ & 28 & 30 & 93.33 & 0 & Normal & CONTROL \\
\hline BBN_20012 & 87 & $\mathrm{~F}$ & 29 & 30 & 96.67 & 0 & Normal & CONTROL \\
\hline BBN_20194 & 102 & $\mathrm{~F}$ & 29 & 30 & 96.67 & 0 & Normal & CONTROL \\
\hline BBN_16210 & 89 & $\mathrm{~F}$ & 29 & 30 & 96.67 & 0 & Normal & CONTROL \\
\hline BBN_9948 & 86 & $\mathrm{~F}$ & 29 & 30 & 96.67 & 0 & Normal & CONTROL \\
\hline BBN_11073 & 90 & $\mathrm{~F}$ & 29 & 30 & 96.67 & 0 & Normal & CONTROL \\
\hline BBN_25743 & 73 & $\mathrm{~F}$ & 29 & 30 & 96.67 & 0 & Normal & CONTROL \\
\hline BBN_4578 & 90 & $\mathrm{~F}$ & 30 & 30 & 100.00 & 0 & Normal & CONTROL \\
\hline BBN_25987 & 98 & $\mathrm{~F}$ & 30 & 30 & 100.00 & 0 & Normal & CONTROL \\
\hline BBN_24243 & 69 & $\mathrm{~F}$ & 30 & 30 & 100.00 & 0 & Normal & CONTROL \\
\hline BBN_16233 & 88 & $\mathrm{~F}$ & 30 & 30 & 100.00 & 0 & Normal & CONTROL \\
\hline BBN_16217 & 85 & $\mathrm{~F}$ & 30 & 30 & 100.00 & 0 & Normal & CONTROL \\
\hline BBN002.29498 & 75 & $\mathrm{~F}$ & 30 & 30 & 100.00 & 0 & Normal & CONTROL \\
\hline BBN_24215 & 91 & $\mathrm{~F}$ & 30 & 30 & 100.00 & 0 & Normal & CONTROL \\
\hline BBN002.28350 & 75 & M & 25 & 30 & 83.33 & 0 & Normal & CONTROL \\
\hline BBN_18399 & 77 & M & 26 & 30 & 86.67 & 0 & Normal & CONTROL \\
\hline BBN_23398 & 84 & M & 26 & 30 & 86.67 & 0 & Normal & CONTROL \\
\hline BBN002.26065 & 74 & M & 27 & 30 & 90.00 & 0 & Normal & CONTROL \\
\hline BBN_10593 & 86 & M & 28 & 30 & 93.33 & 0 & Normal & CONTROL \\
\hline BBN_25741 & 87 & M & 28 & 30 & 93.33 & 0 & Normal & CONTROL \\
\hline BBN002.26311 & 81 & M & 28 & 30 & 93.33 & 0 & Normal & CONTROL \\
\hline BBN002.28858 & 80 & M & 28 & 30 & 93.33 & 0 & Normal & CONTROL \\
\hline BBN_19209 & 82 & M & 28 & 30 & 93.33 & 0 & Normal & CONTROL \\
\hline BBN_4582 & 95 & M & 29 & 30 & 96.67 & 0 & Normal & CONTROL \\
\hline BBN002.26151 & 83 & M & 29 & 30 & 96.67 & 0 & Normal & CONTROL \\
\hline BBN002.29089 & 81 & M & 29 & 30 & 96.67 & 0 & Normal & CONTROL \\
\hline BBN_13802 & 74 & M & 29 & 30 & 96.67 & 0 & Normal & CONTROL \\
\hline BBN002.28121 & 80 & M & 29 & 30 & 96.67 & 0 & Normal & CONTROL \\
\hline BBN002.29416 & 88 & M & 29 & 30 & 96.67 & 0 & Normal & CONTROL \\
\hline BBN_24576 & 92 & M & 29 & 30 & 96.67 & 0 & Normal & CONTROL \\
\hline BBN_10204 & 87 & M & 29 & 30 & 96.67 & 0 & Normal & CONTROL \\
\hline BBN_16213 & 74 & M & 30 & 30 & 100.00 & 0 & Normal & CONTROL \\
\hline BBN_10610 & 78 & M & 30 & 30 & 100.00 & 0 & Normal & CONTROL \\
\hline BBN002.28769 & 91 & M & 25 & 29 & 86.21 & 0 & Normal & $\mathrm{AD}$ \\
\hline BBN_16230 & 80 & $\mathrm{~F}$ & 26 & 30 & 86.67 & 0 & Normal & $\mathrm{AD}$ \\
\hline BBN_15199 & 89 & $\mathrm{~F}$ & 28 & 30 & 93.33 & 0 & Normal & $\mathrm{AD}$ \\
\hline BBN002.29617 & 83 & M & 26 & 30 & 86.67 & 0 & Normal & $\mathrm{AD}$ \\
\hline BBN_16231 & 83 & M & 25 & 30 & 83.33 & 0 & Normal & FTD \\
\hline BBN002.29836 & 84 & $\mathrm{~F}$ & 28 & 30 & 93.33 & 0 & Normal & CVD \\
\hline BBN_24680 & 82 & M & 26 & 30 & 86.67 & 0 & Normal & CVD \\
\hline BBN_20618 & 86 & $\mathrm{~F}$ & 30 & 30 & 100.00 & 0 & Normal & $\mathrm{AD}+\mathrm{CVD}$ \\
\hline
\end{tabular}


Table 5 (continued)

\begin{tabular}{|c|c|c|c|c|c|c|c|c|}
\hline MRC ID & Age at death & Gender & MMSE score & MMSE out of & $\begin{array}{l}\text { Percentage } \\
\text { score }(\%)\end{array}$ & $\begin{array}{l}\text { Coded } \\
\text { MMSE }\end{array}$ & $\begin{array}{l}\text { Impairment of } \\
\text { cognitive func- } \\
\text { tioning }\end{array}$ & Clinical Diagnosis Group \\
\hline BBN002.29535 & 83 & M & 25 & 30 & 83.33 & 0 & Normal & $\mathrm{AD}+\mathrm{DLB}$ \\
\hline BBN_10599 & 85 & $\mathrm{~F}$ & 28 & 30 & 93.33 & 0 & Normal & Other/unspecified \\
\hline BBN_19696 & 93 & M & 28 & 30 & 93.33 & 0 & Normal & Other/unspecified \\
\hline BBN_16199 & 85 & M & 15 & 21 & 71.43 & 1 & Mild & CONTROL \\
\hline BBN_10611 & 92 & $\mathrm{~F}$ & 17 & 24 & 70.83 & 1 & Mild & CONTROL \\
\hline BBN002.29843 & 103 & $\mathrm{~F}$ & 20 & 27 & 74.07 & 1 & Mild & CONTROL \\
\hline BBN_18804 & 88 & M & 23 & 30 & 76.67 & 1 & Mild & $\mathrm{AD}$ \\
\hline BBN002.26278 & 74 & M & 24 & 30 & 80.00 & 1 & Mild & $\mathrm{AD}$ \\
\hline BBN002.28945 & 81 & $\mathrm{~F}$ & 21 & 30 & 70.00 & 1 & Mild & CVD \\
\hline BBN002.28123 & 87 & M & 21 & 30 & 70.00 & 1 & Mild & DLB \\
\hline BBN_10191 & 84 & M & 22 & 30 & 73.33 & 1 & Mild & Other/unspecified \\
\hline BBN_24246 & 91 & $\mathrm{~F}$ & 13 & 30 & 43.33 & 2 & Moderate & CONTROL \\
\hline BBN002.29910 & 68 & $\mathrm{~F}$ & 11 & 30 & 36.67 & 2 & Moderate & $\mathrm{AD}$ \\
\hline BBN_9919 & 91 & $\mathrm{~F}$ & 13 & 30 & 43.33 & 2 & Moderate & $\mathrm{AD}$ \\
\hline BBN_25890 & 76 & $\mathrm{~F}$ & 14 & 30 & 46.67 & 2 & Moderate & $\mathrm{AD}$ \\
\hline BBN002.29132 & 90 & $\mathrm{~F}$ & 14 & 30 & 46.67 & 2 & Moderate & $\mathrm{AD}$ \\
\hline BBN_9944 & 84 & $\mathrm{~F}$ & 15 & 30 & 50.00 & 2 & Moderate & $\mathrm{AD}$ \\
\hline BBN_10598 & 90 & $\mathrm{~F}$ & 16 & 30 & 53.33 & 2 & Moderate & $\mathrm{AD}$ \\
\hline BBN_15195 & 84 & $\mathrm{~F}$ & 18 & 30 & 60.00 & 2 & Moderate & $\mathrm{AD}$ \\
\hline BBN002.28925 & 87 & M & 10 & 30 & 33.33 & 2 & Moderate & $\mathrm{AD}$ \\
\hline BBN_21800 & 82 & M & 11 & 30 & 36.67 & 2 & Moderate & $\mathrm{AD}$ \\
\hline BBN002.28543 & 96 & M & 12 & 30 & 40.00 & 2 & Moderate & $\mathrm{AD}$ \\
\hline BBN_9900 & 88 & M & 17 & 30 & 56.67 & 2 & Moderate & $\mathrm{AD}$ \\
\hline BBN_18800 & 90 & M & 18 & 30 & 60.00 & 2 & Moderate & $\mathrm{AD}$ \\
\hline BBN_21792 & 87 & M & 19 & 30 & 63.33 & 2 & Moderate & $\mathrm{AD}$ \\
\hline BBN002.29842 & 82 & M & 19 & 30 & 63.33 & 2 & Moderate & $\mathrm{AD}$ \\
\hline BBN_9938 & 72 & M & 11 & 30 & 36.67 & 2 & Moderate & FTD \\
\hline BBN_16229 & 79 & M & 18 & 30 & 60.00 & 2 & Moderate & CVD \\
\hline BBN002.28665 & 86 & M & 18 & 30 & 60.00 & 2 & Moderate & CVD \\
\hline BBN_9918 & 88 & $\mathrm{~F}$ & 16 & 30 & 53.33 & 2 & Moderate & $\mathrm{AD}+\mathrm{CVD}$ \\
\hline BBN_24369 & 91 & $\mathrm{~F}$ & 18 & 30 & 60.00 & 2 & Moderate & $\mathrm{AD}+\mathrm{CVD}$ \\
\hline BBN_20991 & 83 & M & 11 & 30 & 36.67 & 2 & Moderate & DLB \\
\hline BBN_9940 & 67 & M & 20 & 30 & 66.67 & 2 & Moderate & other/unspecified \\
\hline BBN_9984 & 79 & M & 0 & 16 & 0.00 & 3 & Severe & $\mathrm{AD}$ \\
\hline BBN_24356 & 80 & $\mathrm{~F}$ & 5 & 20 & 25.00 & 3 & Severe & $\mathrm{AD}$ \\
\hline BBN002.26315 & 93 & $\mathrm{~F}$ & 0 & 28 & 0.00 & 3 & Severe & $\mathrm{AD}$ \\
\hline BBN_16234 & 88 & $\mathrm{~F}$ & 0 & 30 & 0.00 & 3 & Severe & $\mathrm{AD}$ \\
\hline BBN_15196 & 94 & $\mathrm{~F}$ & 0 & 30 & 0.00 & 3 & Severe & $\mathrm{AD}$ \\
\hline BBN_9981 & 95 & $\mathrm{~F}$ & 0 & 30 & 0.00 & 3 & Severe & $\mathrm{AD}$ \\
\hline BBN002.29877 & 99 & $\mathrm{~F}$ & 0 & 30 & 0.00 & 3 & Severe & $\mathrm{AD}$ \\
\hline BBN_24938 & 87 & $\mathrm{~F}$ & 0 & 30 & 0.00 & 3 & Severe & $\mathrm{AD}$ \\
\hline BBN_18400 & 94 & $\mathrm{~F}$ & 0 & 30 & 0.00 & 3 & Severe & $\mathrm{AD}$ \\
\hline BBN_22595 & 94 & $\mathrm{~F}$ & 0 & 30 & 0.00 & 3 & Severe & $\mathrm{AD}$ \\
\hline BBN_16226 & 91 & $\mathrm{~F}$ & 0 & 30 & 0.00 & 3 & Severe & $\mathrm{AD}$ \\
\hline BBN_19999 & 77 & $\mathrm{~F}$ & 0 & 30 & 0.00 & 3 & Severe & $\mathrm{AD}$ \\
\hline BBN_15301 & 87 & $\mathrm{~F}$ & 2 & 30 & 6.67 & 3 & Severe & $\mathrm{AD}$ \\
\hline BBN_14413 & 98 & $\mathrm{~F}$ & 7 & 30 & 23.33 & 3 & Severe & $\mathrm{AD}$ \\
\hline BBN_20621 & 89 & M & 0 & 30 & 0.00 & 3 & Severe & $\mathrm{AD}$ \\
\hline
\end{tabular}


Table 5 (continued)

\begin{tabular}{|c|c|c|c|c|c|c|c|c|}
\hline MRC ID & Age at death & Gender & MMSE score & MMSE out of & $\begin{array}{l}\text { Percentage } \\
\text { score }(\%)\end{array}$ & $\begin{array}{l}\text { Coded } \\
\text { MMSE }\end{array}$ & $\begin{array}{l}\text { Impairment of } \\
\text { cognitive func- } \\
\text { tioning }\end{array}$ & Clinical Diagnosis Group \\
\hline BBN_24944 & 81 & M & 0 & 30 & 0.00 & 3 & Severe & $\mathrm{AD}$ \\
\hline BBN_25642 & 95 & M & 0 & 30 & 0.00 & 3 & Severe & $\mathrm{AD}$ \\
\hline BBN_23391 & 84 & M & 0 & 30 & 0.00 & 3 & Severe & $\mathrm{AD}$ \\
\hline BBN_20018 & 83 & M & 0 & 30 & 0.00 & 3 & Severe & $\mathrm{AD}$ \\
\hline BBN_16228 & 96 & M & 0 & 30 & 0.00 & 3 & Severe & $\mathrm{AD}$ \\
\hline BBN_20003 & 62 & M & 0 & 30 & 0.00 & 3 & Severe & $\mathrm{AD}$ \\
\hline BBN_9929 & 70 & M & 0 & 30 & 0.00 & 3 & Severe & $\mathrm{AD}$ \\
\hline BBN_24939 & 86 & M & 0 & 30 & 0.00 & 3 & Severe & $\mathrm{AD}$ \\
\hline BBN_16207 & 88 & M & 0 & 30 & 0.00 & 3 & Severe & $\mathrm{AD}$ \\
\hline BBN_15304 & 80 & M & 0 & 30 & 0.00 & 3 & Severe & $\mathrm{AD}$ \\
\hline BBN002.29900 & 86 & M & 0 & 30 & 0.00 & 3 & Severe & $\mathrm{AD}$ \\
\hline BBN002.29053 & 71 & M & 2 & 30 & 6.67 & 3 & Severe & $\mathrm{AD}$ \\
\hline BBN_9972 & 92 & M & 7 & 30 & 23.33 & 3 & Severe & $\mathrm{AD}$ \\
\hline BBN002.29071 & 94 & M & 7 & 30 & 23.33 & 3 & Severe & $\mathrm{AD}$ \\
\hline BBN_24550 & 85 & M & 8 & 30 & 26.67 & 3 & Severe & $\mathrm{AD}$ \\
\hline BBN_13796 & 81 & M & 8 & 30 & 26.67 & 3 & Severe & $\mathrm{AD}$ \\
\hline BBN_24199 & 84 & M & 9 & 30 & 30.00 & 3 & Severe & $\mathrm{AD}$ \\
\hline BBN002.26717 & 70 & $\mathrm{~F}$ & 0 & 30 & 0.00 & 3 & Severe & FTD \\
\hline BBN_24551 & 74 & $\mathrm{M}$ & 0 & 30 & 0.00 & 3 & Severe & FTD \\
\hline BBN002.29499 & 74 & M & 0 & 30 & 0.00 & 3 & Severe & FTD \\
\hline BBN_10206 & 87 & $\mathrm{~F}$ & 4 & 30 & 13.33 & 3 & Severe & CVD \\
\hline BBN002.28117 & 95 & $\mathrm{~F}$ & 8 & 30 & 26.67 & 3 & Severe & CVD \\
\hline BBN_19996 & 76 & $\mathrm{~F}$ & 3 & 25 & 12.00 & 3 & Severe & $\mathrm{AD}+\mathrm{CVD}$ \\
\hline BBN_24202 & 86 & $\mathrm{~F}$ & 0 & 30 & 0.00 & 3 & Severe & $\mathrm{AD}+\mathrm{CVD}$ \\
\hline BBN002.29634 & 73 & M & 0 & 30 & 0.00 & 3 & Severe & $\mathrm{AD}+\mathrm{CVD}$ \\
\hline BBN002.28700 & 74 & M & 2 & 16 & 12.50 & 3 & Severe & $\mathrm{AD}+\mathrm{DLB}$ \\
\hline BBN002.29411 & 80 & M & 0 & 30 & 0.00 & 3 & Severe & $\mathrm{AD}+\mathrm{DLB}$ \\
\hline
\end{tabular}

Table 6 Spread of the most recent MMSE scores between diagnosis groups

\begin{tabular}{lllllllc}
\hline $\begin{array}{l}\text { Clinical } \\
\text { diagnosis } \\
\text { group }\end{array}$ & $\begin{array}{l}\text { Mean } \\
\text { MMSE } \\
\text { score }\end{array}$ & $\begin{array}{l}\text { Mean } \\
\text { percentage } \\
\text { score }\end{array}$ & $\begin{array}{l}\text { Number } \\
\text { of cases }\end{array}$ & $\begin{array}{l}\text { Normal } \\
\text { cognitive state } \\
25-30\end{array}$ & $\begin{array}{l}\text { Mild impairment of } \\
\text { cognitive functioning } \\
21-24\end{array}$ & $\begin{array}{l}\text { Moderate impairment } \\
\text { of cognitive functioning } \\
10-20\end{array}$ & $\begin{array}{l}\text { Severe impairment of } \\
\text { cognitive function- } \\
\text { ing }<9\end{array}$ \\
\hline Control & $27 / 30$ & 90.0 & 44 & 40 & 3 & 1 & 0 \\
AD & $8 / 29$ & 27.6 & 52 & 4 & 2 & 14 & 32 \\
FTD & $7 / 30$ & 23.3 & 5 & 1 & 0 & 2 & 3 \\
CVD & $18 / 30$ & 60.0 & 7 & 2 & 1 & 2 & 2 \\
AD+CVD & $11 / 29$ & 37.9 & 6 & 1 & 0 & 1 & 3 \\
DLB & $16 / 30$ & 53.3 & 2 & 0 & 1 & 0 & 0 \\
AD+DLB & $9 / 25$ & 36.0 & 3 & 1 & 0 & 1 & 2 \\
Other & $25 / 30$ & 83.3 & 4 & 2 & 1 & 22 & 0 \\
Total & & & 123 & 51 & 8 & & 42 \\
\hline
\end{tabular}

thought to be normal aging changes; therefore, we considered these mismatched cases. Someone with moderate AD pathology may or may not present with clinical symptoms of dementia thus making it difficult for a GP or care giver to recognise. Subtle cognitive changes may be missed by the standardised clinical assessments.

Findings from the 'Nun Study of Aging and Alzheimer's Disease' also suggests that pathology can be present 
in the absence of clinical symptom presentation (Snowdon 1997). This continuing longitudinal study produced clinical and neuropathological data on 498 Catholic sisters, who all lived in relative homogeneity in terms of environment and lifestyle. $12 \%$ of participants who were not demented were found to have Braak stages V-VI pathology (SantaCruz 2011). An especially interesting case of an 85 years with well-preserved cognitive and physical function with a genetic predisposition to Alzheimer's disease and an abundance of Alzheimer's disease lesions was found (Snowdon 1997). This study provides useful information on how the degree of pathology present in the brain and the level of resistance to the clinical expression of the neuropathology can occur in some cases.

The nun study also found $29 \%$ of participants with dementia exhibited Braak stage II pathology or less (SantaCruz 2011). In the BDR cohort one case was diagnosed clinically as AD and presented symptoms of $\mathrm{AD}$, such as significant problems in memory, irritable behavior and personality changes. Additionally, the brain scans indicated frontal lobe shrinkage. However, the only neuropathology observed in this case was minimal AD pathology Braak stage II and sparse AGD (argyrophilic grain disease) pathology. The microscopy report noted that it was difficult to explain the cognitive decline with the pathology presented. This is rare, however, not unheard of.

Nolan et al. (2015) demonstrated the importance of thorough neuropathological assessment on control tissue. This study re-investigated tissue donated prior to 2007, when new histological staining protocols for examining control brain were introduced. Almost all cases that were originally described as neuropathologically normal displayed some level of pathology after re-analysis. Four cases displayed 'major' pathological features which are usually associated with significant clinical features of dementia. This finding emphasises the importance of accurate neuropathological analysis of control tissue and highlights the inherent difficulty of classifying tissue as 'control'.

In the clinical AD group 37\% did not directly match pathologically. The majority of these cases had Alzheimer's disease pathology but with additional prominent DLB or CVD pathology (fulfilling a combined diagnosis). This suggests that on the clinical side, diagnostic teams may not be detecting additional 'DLB' and 'CVD' clinical symptoms via the various assessment measures. The symptoms occurring in CVD, such as unusual movements, falls, problems with planning and organising, decision making or problem solving were not picked up by the current assessment methods (Jellinger 2008). Symptoms such as memory loss or confusion, which are more commonly associated with $\mathrm{AD}$, are better addressed in the assessments. Both these symptoms are also commonly seen in CVD, which does make distinguishing CVD from
AD difficult for assessors and GPs. With AD being the most common type of dementia, it seems GPs and assessors are more likely to diagnose people with AD. Brenowitz et al. (2017a, b) investigated whether associations between clinical progression and AD neuropathology were modified by co-occurring Lewy body disease (LBD). They found overall AD + LBD was associated with faster rate of progression than $\mathrm{AD}$ only, it was most evident in cases with intermediate AD pathology. Patients with DLB also have additional clinical symptoms such as hallucinations and parkinsonism and a worse prognosis than pure AD (Mueller 2017) and, therefore, often require specific management.

Our results are reflective of data collected from nine brain bank centres within the BrainNet Europe consortium (3303 cases) which showed $53.3 \%$ of dementia cases had a mixed pathological diagnosis (Kovacs 2008). This correlates with a number of other studies emphasising the contribution of mixed brain pathologies to dementia cases (Kovacs 2013; Mendez 1992; Robinson 2018).

Monitoring cognitive decline from an early point and applying diagnostic procedures to increase detection of dementias other than AD may highlight those who may benefit from treatments targeting multiple aetiologies.

The 'CVD' clinical group was poorly matched, only one case was a complete match clinically and pathologically. Three out of ten in this group had AD + CVD mixed pathology. The 'AD + CVD' clinical group had most of the cases mismatching, only 5 of the 19 in this group were a complete match. 6 of the 19 were pathologically diagnosed with $\mathrm{AD}$ in the absence of additional 'CVD' pathology. We utilised VCING scores to determine the level of vascular involvement in contributing to the dementia, we also looked at past medical records to see if any vascular changes were clinically observed and recorded. Seven cases in the group had no vascular neuropathology at all. This would suggest clinicians may be over-diagnosing patients with mixed AD and vascular dementia; when there is no clinical or pathological evidence of a vascular component. Brunnstrom et al. (2009) found similarly poorly matched CVD (59\%) and mixed $\mathrm{AD}+\mathrm{CVD}(25 \%)$ cases in their clinicopathological concordance study in Sweden. This is important, since it may lead to these patients not being treated with anti-dementia drugs such as ACE and memantine.

We can conclude that the clinical assessment procedures are not designed to pick up on an additional 'CVD' or 'DLB' component of $\mathrm{AD}$ type dementia (whereas CVD on its own is over-diagnosed). Statistical analysis showed there was no significant difference in the clinical assessment scores between the matched and mismatched cases. This could be due to AD symptoms masking the additional presentation of 'CVD' and 'DLB' symptoms, therefore, not being picked up efficiently during the diagnosis and assessment. When 
dementia patients are in the last stages of the dementia, quite often the symptoms presented are severe cognitive decline and memory loss, and inability to take care of oneself. If a patient is only diagnosed during this late stage, assessors may have missed other possible clinical presentations such as hallucinations, apathy and psychiatric syndromes, which may have eluded to the subtype of dementia.

In the clinical FTD group, four out of six cases were pathologically diagnosed as having FTLD. Correct identification of clinical 'red flags' for FTD may be responsible for the relatively high number of matched cases in the group. Behavioral or personality changes in the absence of prior psychiatric history often appears first; language decline characterised by impaired speech production or impaired word comprehension and semantic memory is also a key feature of the condition (Warren 2013). Memory, navigational skills and other aspects of general intellect are often well maintained in FTD patients initially. More widespread cognitive deficits like AD phenotypes emerging later in disease course. Hence the importance of early detection of the condition.

Though FTD is substantially less common than AD, the prevalence of FTD in older age groups has been almost certainly been underestimated (Warren 2013). Echavarri et al. (2012) investigated the agreement between clinical and neuropathological diagnoses of dementia cases donated to the Brain Bank of Navarre, Spain and found the agreement in FTLD (28\%) to be much lower than the other dementia subtypes examined. In the BDR cohort we pathologically identified 15 FTLD cases; 7 of these cases were clinically diagnosed with $\mathrm{AD}$ and 2 cases were clinically diagnosed with $\mathrm{AD}+\mathrm{CVD}$. This suggests clinically FTD is being under diagnosed and misdiagnosed as $\mathrm{AD}$ or $\mathrm{AD}+\mathrm{CVD}$. This could be due to patients not being seen until later in the disease progression when symptoms of memory and cognitive decline have emerged and mask the more unique FTD symptoms. New consensus diagnostic criteria for FTD and other progressive aphasias have recently been formulated (Rascovsky 2011). It is important for non-specialists to have a workable framework for suspecting FTD as it can be particularly challenging to diagnose (Rascovsky 2011).

Neuropsychiatric inventory (NPI) scores are taken during BDR clinical assessments; however, over half the BDR cases were missing these scores, suggesting NPIs are not being utilised as much as they could during assessments and resulting in potentially missed cases of FTD and DLB.

Pathologically, the diagnosis for the control, and AD groups were well matched to the clinical diagnosis. This could imply the assessments are best suited in distinguishing controls and AD. A previous study by Beach et al. (2012) looked at the sensitivity and specificity of the clinical diagnosis of $\mathrm{AD}$ against the neuropathological diagnosis, they found sensitivity for clinical diagnosis was increased with more permissive clinical criteria and specificity was increased with more restrictive clinical criteria. The study carried out by the National institute of aging (NIA), for the Alzheimer's Disease Cooperative Study (ADCs), found they were more accurate when they diagnosed $\mathrm{AD}$, among dementia subjects, than when they diagnosed subjects with other dementing diseases which included; AGD, FTLD, CVD and DLB.

These studies have found uncertainties in diagnosing mixed dementia during life which remain a major limitation in advancing research on the relationship between $\mathrm{AD}$ and CVD, limiting the precision of diagnostic decisions.

It is also important to consider that the clinical diagnoses provided by memory services/hospital teams or GPs are not readily available to BDR assessment teams unless disclosed by the next of kin or participant. The BDR assessments occur over an average duration of 8 years; however, missed assessments, and missed questions can be expected due to the nature of the dementing conditions. Additionally, control participants are assessed every 2 years, if they develop a cognitive impairment in those 2 years this may not be picked up by the team.

Future proposals for early detection and diagnosis of dementia patients would increase the likelihood of more accurate diagnosis of dementia subtypes based on observable presentation of DLB, FTD and CVD. The use of more efficient and continued assessments throughout the disease progression would also allow for better care and understanding of the patient based on the dementia subtype.

Acknowledgements The London Neurodegenerative Diseases Brain Bank receives funding from the MRC and as part of the Brains for Dementia Research program, jointly funded by Alzheimer's Research UK and Alzheimer's Society. We thank the staff of the Clinical Neuropathology Department at King's College Hospital for diagnostic and technical assistance and the BDR team at Cardiff University. We also thank the donors and their families.

OpenAccess This article is distributed under the terms of the Creative Commons Attribution 4.0 International License (http://creativeco mmons.org/licenses/by/4.0/), which permits unrestricted use, distribution, and reproduction in any medium, provided you give appropriate credit to the original author(s) and the source, provide a link to the Creative Commons license, and indicate if changes were made.

\section{References}

Alafuzoff I et al (2009) Assessment of beta-amyloid deposits in human brain: a study of the BrainNet Europe Consortium. Acta Neuropathol 117(3):309-320

Beach TG et al (2012) Accuracy of the clinical diagnosis of Alzheimer disease at National Institute on Aging Alzheimer Disease Centers, 2005-2010. J Neuropathol Exp Neurol 71(4):266-273

Braak H et al (2006) Staging of Alzheimer disease-associated neurofibrillary pathology using paraffin sections and immunocytochemistry. Acta Neuropathol 112(4):389-404 
Brenowitz WD et al (2017a) Mixed neuropathologies and estimated rates of clinical progression in a large autopsy sample. Alzheimers Dement 13(6):654-662

Brenowitz WD et al (2017b) Alzheimer's disease neuropathologic change, Lewy body disease, and vascular brain injury in clinicand community-based samples. Neurobiol Aging 53:83-92

Brunnstrom H, Englund E (2009) Clinicopathological concordance in dementia diagnostics. Am J Geriatr Psychiatry 17(8):664-670

Crary JF et al (2014) Primary age-related tauopathy (PART): a common pathology associated with human aging. Acta Neuropathol 128(6):755-766

Echavarri C et al (2012) Co-occurrence of different pathologies in dementia: implications for dementia diagnosis. J Alzheimers Dis 30(4):909-917

Hayes G, Guidelines for BDR assessments. 2016

Jack CR Jr et al (2011) Introduction to the recommendations from the National Institute on Aging-Alzheimer's Association workgroups on diagnostic guidelines for Alzheimer's disease. Alzheimers Dement 7(3):257-262

Jellinger KA (2008) The pathology of "vascular dementia": a critical update. J Alzheimers Dis 14(1):107-123

Kovacs GG et al (2008) Mixed brain pathologies in dementia: the BrainNet Europe consortium experience. Dement Geriatr Cogn Disord 26(4):343-350

Kovacs GG et al (2013) Non-Alzheimer neurodegenerative pathologies and their combinations are more frequent than commonly believed in the elderly brain: a community-based autopsy series. Acta Neuropathol 126(3):365-384

McKeith IG et al (2005) Diagnosis and management of dementia with Lewy bodies: third report of the DLB consortium. Neurology 65(12):1863-1872

Mendez MF et al (1992) Clinically diagnosed Alzheimer disease: neuropathologic findings in 650 cases. Alzheimer Dis Assoc Disord 6(1):35-43

Mueller C et al (2017) The prognosis of dementia with Lewy bodies. Lancet Neurol 16(5):390-398

NICE (2001) National Institute for Clinical Excellence. Guidance on the use of donepezil, rivastigmine and galantamine for the treatment of Alzheimer's disease. https://www.nice.org.uk/guidance/ ta111/documents/alzheimers-disease-donepezil-rivastigminegalantamine-and-memantine-review-final-appraisal-document2. Accessed 24 Aug 2017
NICE (2006) National Institute for Health and Care Excellence: guidance diagnosis and assessment of dementia. https://www.nice. org.uk/guidance/CG42/chapter/1-Guidance\#diagnosis-and-asses sment-of-dementia. Accessed 24 Aug 2017

NICE (2018) National Institute for Health and Care Excellence: dementia: assessment, management and support for people living with dementia and their carers. https://www.nice.org.uk/guidance/ ng97. Accessed 6 Dec 2018

Nolan M et al (2015) Control tissue in brain banking: the importance of thorough neuropathological assessment. J Neural Transm (Vienna) 122(7):949-956

Price JL et al (2009) Neuropathology of nondemented aging: presumptive evidence for preclinical Alzheimer disease. Neurobiol Aging 30(7):1026-1036

Rascovsky K et al (2011) Sensitivity of revised diagnostic criteria for the behavioural variant of frontotemporal dementia. Brain $134(\mathrm{Pt}$ 9):2456-2477

Robinson JL et al (2018) Neurodegenerative disease concomitant proteinopathies are prevalent, age-related and APOE4-associated. Brain 141(7):2181-2193

SantaCruz KS et al (2011) Alzheimer disease pathology in subjects without dementia in 2 studies of aging: the Nun Study and the Adult Changes in Thought Study. J Neuropathol Exp Neurol $70(10): 832-840$

Skrobot OA et al. (2016) Vascular cognitive impairment neuropathology guidelines (VCING): the contribution of cerebrovascular pathology to cognitive impairment. Brain 139:2957-2969

Snowdon DA (1997) Aging and Alzheimer's disease: lessons from the Nun Study. Gerontologist 37(2):150-156

Sperling RA et al (2011) Toward defining the preclinical stages of Alzheimer's disease: recommendations from the National Institute on Aging-Alzheimer's Association workgroups on diagnostic guidelines for Alzheimer's disease. Alzheimers Dement 7(3):280-292

Warren JD, Rohrer JD, Rossor MN (2013) Clinical review. Frontotemporal dementia. BMJ 347:f4827

Alzheimer's Disease International World Alzheimer Report (2011) The benefits of early diagnosis and intervention. [Report] 2011. https:// www.alz.co.uk/research/WorldAlzheimerReport2011.pdf

Publisher's Note Springer Nature remains neutral with regard to jurisdictional claims in published maps and institutional affiliations. 